\title{
DEPREDACIÓN POR PODISUS MACULIVENTRIS (SAY) SOBRE LARVAS DE CHORISTONEURA ROSACEANA (HARRIS)
}

\author{
Daniela NÁJERA-MIRAMONTES, ${ }^{1}$ MAgali ORDÓÑEZ-GARCÍA, ${ }^{1}$ Claudio RÍOS-VELASCO, ${ }^{2, *}$ DaVID I. \\ BERlangA-REYeS, ${ }^{2}$ Carlos H. ACOSTA-MUÑIZ, ${ }^{2}$ Paul B. ZAMUdiO-FlORES, ${ }^{2}$ Alejandro ROMO- \\ CHACÓN ${ }^{2}$ y JUAN L. JACOBO-CUÉLLAR ${ }^{3}$
}

\author{
${ }^{1}$ Estudiantes de Maestría en Ciencias, Centro de Investigación en Alimentación y Desarrollo, A.C., Unidad \\ Cuauhtémoc, Chihuahua. Av. Río Conchos S/N, Parque Industrial, C.P. 31570, Cuauhtémoc, Chihuahua, México. \\ ${ }^{2}$ Centro de Investigación en Alimentación y Desarrollo, A.C., Unidad Cuauhtémoc, Chihuahua, Av. Río Conchos \\ S/N, Parque Industrial, C.P. 31570, Cuauhtémoc, Chihuahua, México. \\ ${ }^{3}$ Campo Experimental de la Sierra de Chihuahua, Instituto Nacional de Investigaciones Forestales, Agrícolas y \\ Pecuarias. Hidalgo 1213, Zona Centro, C.P. 31500, Cuauhtémoc, Chihuahua, México. \\ *Autor de correspondencia: <claudio.rios@ciad.mx>
}

Recibido: 20/02/2015; aceptado: 02/03/2016

Nájera-Miramontes, D., Ordóñez-García, M., Ríos-Velasco, C., Berlanga-Reyes, D. I., Acosta-Muñiz, C. H., Zamudio-Flores, P. B., Romo-Chacón, A. \& Jacobo-Cuéllar, J. L. 2016. Depredación por Podisus maculiventris (Say) sobre larvas de Choristoneura rosaceana (Harris). Acta Zoológica Mexicana (n. s.), 32(2): 147-152.

RESUMEN. Choristoneura rosaceana (Harris) es una plaga del manzano Malus domestica Borkh. de reciente aparición en México. Su control depende básicamente del uso de insecticidas químicos; sin embargo, existen registros de enemigos naturales de esta plaga que ejercen un control considerable. El objetivo del estudio fue evaluar la capacidad de depredación de Podisus maculiventris (Say) sobre C. rosaceana. Para lo anterior, en agosto y septiembre de 2014, se recolectaron 1200 larvas de C. rosaceana en huertos de manzano. Asimismo, se recolectaron 87 especímenes del depredador $P$. maculiventris, de diferentes estadios ninfales, de los cuales 75 se recolectaron en huertos de manzano y 17 en parcelas de maíz, además se recolectaron 22 grupos de huevos (17 en maíz y cinco en manzano). Se realizaron ensayos de consumo de $P$. maculiventris sobre larvas de $C$. rosaceana, donde el promedio fue de 0.72 larvas consumidas por día, siendo el primer estadio ninfal el que mostró un mayor promedio de consumo (1.9 larvas/día), en las primeras 24 h y el segundo estadio mostró el mayor promedio a los 7 días (1.2 larvas consumidas/día).

Palabras clave: Control biológico, chinche soldado, depredador, entomófago, enrollador de la hoja.

\section{INTRODUCCIÓN}

Choristoneura rosaceana (Harris) (Lepidoptera: Tortricidae) es un fitófago de reciente introducción en la región manzanera del estado de Chihuahua, México, ya que el primer reporte de su presencia fue hecho por Bautista et al. (2011), en el municipio de Cuauhtémoc, Chihuahua, donde se estipula un estatus fitosanitario de distribución
Nájera-Miramontes, D., Ordóñez-García, M., Ríos-Velasco, C., Berlanga-Reyes, D. I., Acosta-Muñiz, C. H., Zamudio-Flores, P. B., Romo-Chacón, A. \& Jacobo-Cuéllar, J. L. 2016. Predation by Podisus maculiventris (Say) on Choristoneura rosaceana (Harris) larvae. Acta Zoológica Mexicana (n. s.), 32(2): 147-152.

ABSTRACT. Choristoneura rosaceana (Harris) is an emerging pest of apple Malus domestica Borkh., in Mexico. Its control depends mainly on the use of chemical insecticides; however, there are reports of natural enemies of this pest that exert a considerable control. The main objective of study was to evaluate the predatory capacity by Podisus maculiventris (Say) on C. rosaceana larvae. Twelve hundred larvae of $C$. rosaceana were collected in apple orchards. Moreover, 87 specimens of $P$. maculiventris Say of different nymphal instars were collected, of which 75 specimens were collected in apple orchards and 17 in corn crops, and 22 egg groups (17 in corn crops and five in apple trees). Podisus maculiventris consumption bioassays were performed over $C$. rosaceana larvae, where average was 0.72 larvae consumed per day, first nymphal instar showed a higher average (1.9 larvae per day) in first $24 \mathrm{~h}$, and second nymphal instar showed a higher average after $7 \mathrm{~d}$ (1.2 larvae consumed per day).

Key words: Biological control, spined soldier bug, predator, entomophagous, obliquebanded leafroller.

restringida. Las larvas de este insecto se alimentan y refugian en las hojas, además de causar daño al fruto con el cual se afecta el $90 \%$ de su valor comercial. El combate de C. rosaceana depende básicamente del uso de insecticidas químicos, con riesgo de desarrollo de resistencia, que limitaría la producción de alimentos con mayor severidad en países que, como México, carecen del desarrollo de nuevos productos bioinsecticidas (Lagunes et al. 
2009). Además, con el uso inadecuado de insecticidas se ocasiona contaminación ambiental y se elimina la fauna benéfica (Tinoco \& Halperin 1998, Soto 2008).

El manejo de este lepidóptero es complicado por el comportamiento evasivo al mantenerse protegido durante su etapa larval, ya que se refugia en las hojas del manzano al provocar su enrollamiento y la construcción de un cocón de seda dentro de la misma.

Se han reportado parasitoides, tales como Apanteles polychrosidis Viereck, Hemisturmia tortricis Coquillett, Meteorus trachynotus Viereck, Euderus acrobasis (Crawford), Colpoclypeus florus (Walker), Elachertus argissa (Walker), depredadores como Phymata fasciata Gray, Chrysoperla spp., Harmonia axyridis (Pallas) y entomopatógenos como Metarhizium spp., Beauveria spp., Bacillus thuringiensis, entre otros, como enemigos naturales de C. rosaceana (Li \& Fitzpatrick 1999, Vakenti et al. 2001, Sarvary et al. 2007, Sarvary et al. 2010). Sin embargo, en estos trabajos no se hace mención al depredador generalista Podisus spp. (Hemiptera: Pentatomidae) como enemigo natural de este lepidóptero plaga y que puede ser una herramienta potencial como biocontrolador (Cola-Zanuncio et al. 2008, Jones et al. 2009). El objetivo del estudio fue evaluar la capacidad de depredación de $P$. maculiventris sobre $C$. rosaceana.

\section{MATERIAL Y MÉTODOS}

En los meses de agosto y septiembre del 2014 se recolectaron larvas de $C$. rosaceana en un huerto de manzano ubicado en El Terrero, municipio de Namiquipa, Chihuahua (29 18' 84” N; $107^{\circ} 44^{\prime}$ 21” O). Para la recolecta de larvas se revisaron 50 árboles por huerto (10 en cada punto cardinal y 10 en el centro), las larvas recolectadas se trasladaron al laboratorio y se colocaron en contenedores plásticos de 1 oz de capacidad con dieta artificial (Southland Products Incorporated, USA) y se mantuvieron en una cámara de cría a una temperatura de $27 \pm 1{ }^{\circ} \mathrm{C}$, un fotoperiodo de 12:12 (L:O) y una humedad relativa $>70 \%$. Los ejemplares mantenidos en estas condiciones, se revisaron periódicamente hasta la emergencia de adultos de C. rosaceana y/o parasitoides para su montaje e identificación. A partir de los adultos de C. rosaceana se estableció la cría en laboratorio alimentadas con dieta artificial.

Recolecta e identificación de Podisus maculiventris. Se recolectaron ejemplares de $P$. maculiventris (Hemiptera: Pentatomidae) en agosto y septiembre de 2014, en un huerto de manzano y una parcela de maíz ubicados en El Terrero, Namiquipa (29॰ 18' 84” N; $107^{\circ} 44^{\prime}$ 21” O) (Cuadro 1) y se depositaron en contenedores plásticos de $1 \mathrm{oz}$ de capacidad con una larva de $C$. rosaceana o Spodoptera frugiperda (Lepidoptera: Noctuidae), para su trasladado al Laboratorio de Patología Vegetal y Control Biológico del CIAD, Unidad Cuauhtémoc. Los ejemplares recolectados se mantuvieron bajo condiciones de laboratorio antes mencionadas, alimentados con larvas de $C$. rosaceana, S. frugiperda o Tenebrio molitor (Coleoptera: Tenebrionidae), criadas en laboratorio. La identificación de $P$. maculiventris se llevó a cabo mediante claves taxonómicas y pictóricas (Ortega-León 1997, Capinera 2008, Universidad de Florida s/a, Rider 2012). La confirmación de la especie fue hecha por la M.C. Guillermina Ortega Léon (Instituto de Biología, Departamento de Zoología, Universidad Nacional Autónoma de México, Ciudad de México). Ejemplares de $P$. maculiventris se depositaron en la colección de insectos del Instituto de Biología de la UNAM (CNIN).

Evaluación de la capacidad depredadora de Podisus maculiventris. La capacidad de depredación de P. maculiventris sobre larvas de $C$. rosaceana, se evaluó de acuerdo con la metodología de Cola-Zanuncio et al. (2008), para lo cual, los especímenes de los estadios (n1-n4) de $P$. maculiventris se mantuvieron en inanición por 6 h previo a los ensayos. En el primer día se colocaron cinco larvas de $C$. rosaceana de tercer y cuarto estadio por cada espécimen de $P$. maculiventris. En los días posteriores el número de larvas suministradas fue variable. El ensayo se

Cuadro 1. Número de ejemplares de Podisus maculiventris, recolectados en manzano y maíz en El Terrero, Namiquipa, Chihuahua, México, 2014.

\begin{tabular}{|c|c|c|}
\hline Cultivo & Etapa fenológica de desarrollo & Número de ejemplares \\
\hline \multirow[t]{7}{*}{ Manzano } & Ninfa 1 & 44 \\
\hline & Ninfa 2 & 10 \\
\hline & Ninfa 3 & 5 \\
\hline & Ninfa 4 & 5 \\
\hline & Ninfa 5 & 1 \\
\hline & Adulto & 5 \\
\hline & Grupos de Huevos & 5 \\
\hline \multirow[t]{2}{*}{ Maíz } & Adulto & 17 \\
\hline & Grupos de Huevos & 17 \\
\hline \multirow[t]{2}{*}{ Total } & Especímenes & 92 \\
\hline & Grupos de Huevos & 22 \\
\hline
\end{tabular}

Los huevos se contaron como grupos de huevos. 
llevó a cabo en contenedores plásticos de $8 \times 9 \times 3 \mathrm{~cm}$, y se contaron las larvas consumidas cada 24 h por $7 \mathrm{~d}$. El consumo por estadio por día se analizó estadísticamente a través de intervalos de confianza, en los que la decisión fue: consumo igual con $95 \%$ de confianza entre estadios cuando se compartieron entre sí valores de los intervalos de confianza (Clifford \& Taylor 2008).

\section{RESULTADOS}

De los huertos de manzano muestreados se obtuvieron 1200 larvas de C. rosaceana, a las cuales se les realizó la exploración de parasitoides, sin tener éxitos; sin embargo, se encontró al depredador $P$. maculiventris alimentándose de manera natural de larvas de C. rosaceana (Fig. 1a) y $S$. frugiperda (Fig. 1b), en huertos de manzano y parcelas de maíz, respectivamente. Lo cual indica que este depredador podría tener potencial para controlar a otras especies de lepidópteros como $S$. frugiperda.

Recolecta e identificación de Podisus maculiventris. Se recolectaron 70 especímenes de $P$. maculiventris en diferentes etapas de desarrollo y cinco grupos de huevos, con un promedio de 21 huevos por grupo, en huertos de manzano, mientras que en parcelas de maíz se encontraron 17 adultos y 17 grupos de huevos, con un promedio de 22 huevos por grupo (Cuadro 1). Los especímenes presentaron las características morfológicas: proboscis muy gruesa y antenas de un solo color (Fig. 2a), húmeros agudos espinosos (Fig. 2b), la punta del escutelo triangular proyectado hacia atrás entre las alas y es redondeada (Fig. 2c), esterno pálido con pequeñas manchas y último ester- nito abdominal de la hembra con una gran mancha (Fig. 2d) y fémur con un par de puntos anteapicales (Fig. 2e), mismas que de acuerdo con claves taxonómicas y pictóricas (Ortega-León 1997, Capinera 2008, Universidad de Florida s/a, Rider 2012), coincidieron con las descritas para Podisus maculiventris (Say).

Evaluación de la capacidad depredadora de Podisus maculiventris. Se detectaron diferencias significativas en el consumo promedio de larvas de C. rosaceana por estadios ninfales de $P$. maculiventris en un periodo de siete días (Cuadro 2). Las ninfas del segundo estadio del depredador fueron las más voraces, ya que consumieron entre ocho y nueve larvas del tercer o cuarto estadio de $C$. rosaceana, valores que fueron estadísticamente diferentes a los consumidos por ninfas del primero (6.3-7.3) y tercer estadio (5.3-6.7). Mientras que las ninfas del cuarto estadio mostraron menor capacidad de consumo, con valores que fluctuaron entre 3.3 y 4.3 ejemplares por un periodo de siete días (Cuadro 2). De tal forma, que el consumo potencial de larvas de $C$. rosaceana por ninfas del primer a cuarto estadio de P. maculiventris fluctuó entre 23 y 27 ejemplares.

\section{DISCUSIÓN}

La ausencia de parasitoides en las larvas de C. rosacea$n a$, se debió posiblemente a que esta plaga es de reciente aparición en el país, reportada por Bautista-Martínez et al. (2011), aunado a la poca diversidad vegetal cercana al huerto de manzano (Unruh et al. 2012). Sin embargo, se encontró a $P$. maculiventris depredando de manera na-

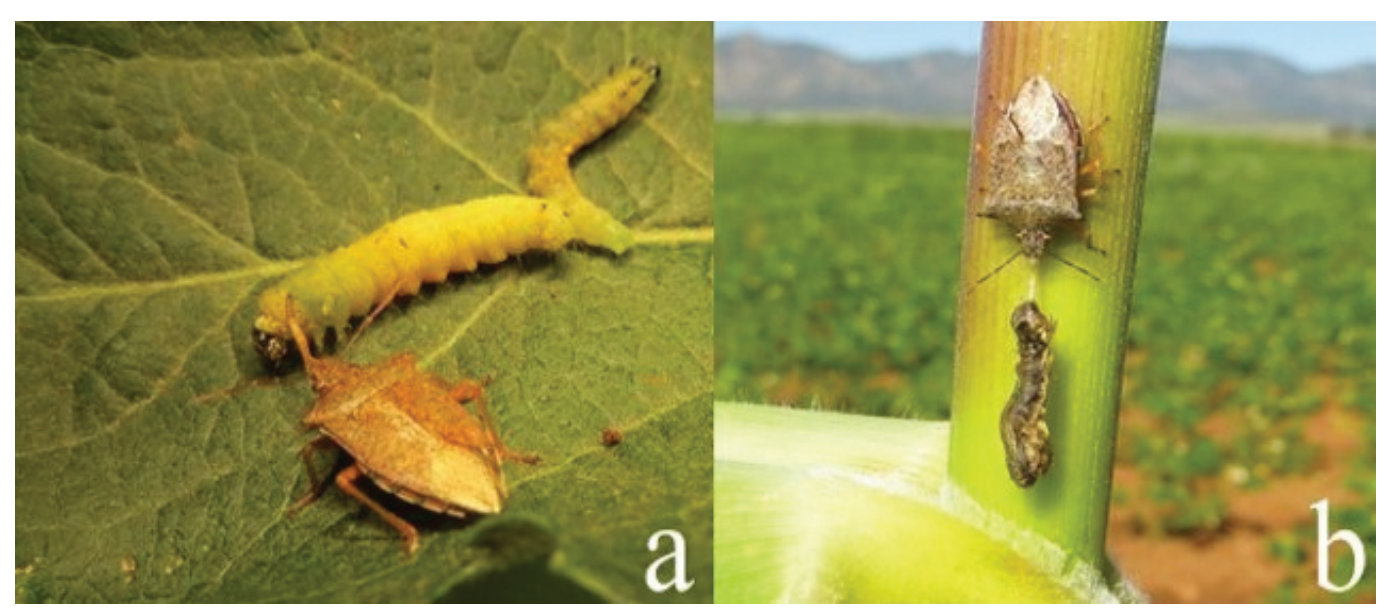

Figura 1. Adultos de Podisus maculiventris, alimentándose de larvas de; a) C. rosaceana y b) S. frugiperda. 


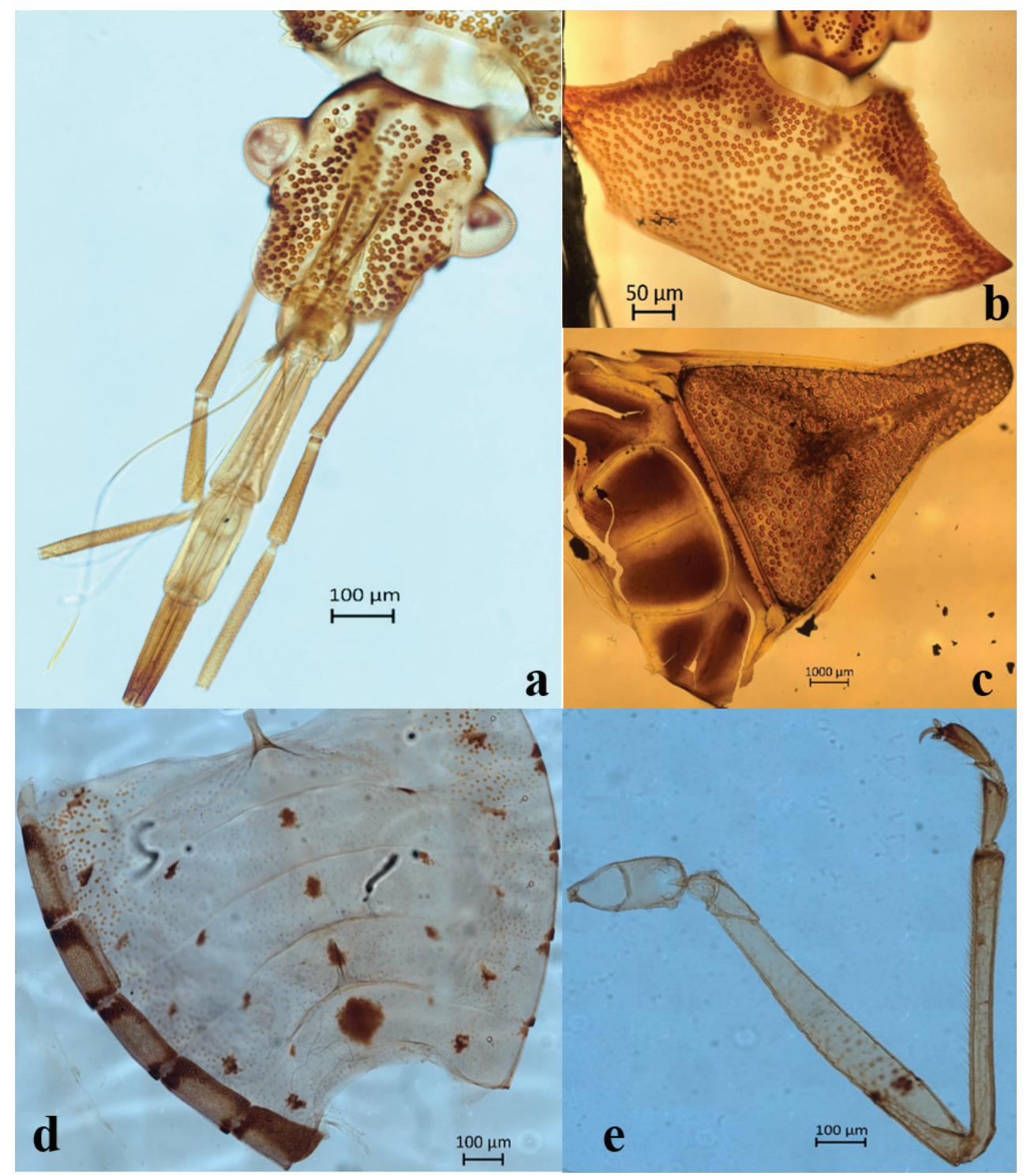

Figura 2. Características morfológicas de Podisus maculiventris; a) región cefálica, b) húmeros agudos espinosos, c) escutelo, d) esterno pálido con pequeñas manchas y último esternito abdominal de la hembra con una gran mancha y e) fémur con un par de puntos anteapicales.

tural a larvas de diferentes estadios de C. rosaceana y $S$. frugiperda, en huertos de manzano y parcelas de maíz, respectivamente. Este depredador ha sido encontrado desde Sudamérica hasta América Central (Ortega-León 1997, Thomas 1992) y es capaz de alimentarse de larvas de más de 90 especies de insectos fitófagos, incluyendo a coleópteros y áfidos, con mayor afinidad a lepidópteros (Torres et al. 2006).

En las regiones agrícolas del estado de Chihuahua no existen reportes de liberación de Podisus spp., por lo que, este insecto se encuentra de manera natural en huertos de manzano y parcelas de maíz depredando larvas de $C$. rosaceana y de $S$. frugiperda, respectivamente, coincidiendo con Ortega-León (1997) quien hace mención de la presencia de $P$. maculiventris en el estado. De Bortoli et al. (2011), Straub et al. (2007) y Barbosa (1998) resaltan la importancia de conservar a estos depredadores en los cultivos ya que proporcionan un control natural de las plagas al mantener la abundancia y diversidad de enemigos naturales, incluyendo la manipulación de hábitats agrícolas a favor de éstos, que permitan su persistencia espacial y temporal. Al respecto, Sarvary et al. (2010) reportan que 
Cuadro 2. Depredación de ninfas de Podisus maculiventris sobre larvas de tercer y cuarto estadio de Choristoneura rosaceana.

\begin{tabular}{|c|c|c|c|c|c|c|c|c|c|}
\hline $\begin{array}{l}\text { Estadio ninfal } \\
\text { del depredador }\end{array}$ & \multicolumn{7}{|c|}{ Consumo promedio de larvas por día } & $\begin{array}{l}\text { Promedio de } \\
\text { consumo por } \\
\text { estadio del } \\
\text { depredador }\end{array}$ & $\begin{array}{c}\text { Intervalo de confianza } \\
\text { para el consumo promedio } \\
\text { de larvas por estadio } \\
\text { ninfal del depredador }\end{array}$ \\
\hline 1 & 1.90 & 1.45 & 0.67 & 0.98 & 0.69 & 0.76 & 0.38 & 0.82 & $6.3-7.3 b$ \\
\hline 2 & 1.8 & 0.9 & 1.5 & 0.8 & 1.1 & 0.8 & 0.8 & 1.1 & $8.0-8.8$ a \\
\hline 4 & 0.2 & 0.2 & 1 & 1 & 0 & 0.2 & 0.2 & 0.4 & $3.3-4.3 c$ \\
\hline Total & 1.06 & 0.73 & 1.09 & 0.79 & 0.54 & 0.49 & 0.39 & 0.72 & \\
\hline \multicolumn{9}{|c|}{ Consumo potencial promedio para cada uno de los estadios ninfales del depredador } & $23.0-27.0$ \\
\hline
\end{tabular}

Valores en la columna de intervalo de confianza con la misma letra significa igualdad estadística entre ellos con 95\% de confianza.

las generaciones de verano de $C$. rosaceana son escasas en hábitats no perturbados, pero abundantes en huertos de manzano manejados y lo atribuye a un mayor parasitismo o depredación en los primeros, lo cual sugiere la alta susceptibilidad de los parasitoides y depredadores al manejo convencional de los sistemas agrícolas.

Con respecto a la capacidad de depredación de P. maculiventris sobre larvas de C. rosaceana, Cola-Zanuncio et al. (2008) mencionan que a mayor densidad de larvas de la presa, el porcentaje de depredación es mayor por parte de $P$. maculiventris. El consumo promedio de larvas de $C$. rosaceana de los estadios ninfales 3 y 4 fue menor, debido a que hubo una menor disponibilidad de presas (Cola-Zanuncio et al. 2008). Vivan et al. (2003) observaron que la capacidad depredadora de P. nigrispinus (Dallas) no se ve afectada por el ambiente donde se encuentra, sin embargo, el consumo sí se ve afectado por el tipo de presa disponible.

El empleo de este depredador como agente de control biológico de plagas, es recomendable, por su amplio rango de presas, capacidad depredadora, capacidad de búsqueda, fácil manejo en laboratorio y liberación en campo, y de adaptación a sistemas agrícolas y condiciones agroclimáticas, por lo que, se recomienda implementar y/o fortalecer el control biológico por conservación dado que este depredador se encuentra de manera natural en las regiones agrícolas del estado.

AGRADECIMIENTOS. Expresamos nuestro sincero agradecimiento a la Fundación Produce Chihuahua, A.C., por el apoyo financiero del proyecto, con número de registro 499. La autora Daniela Nájera Miramontes, agradece al Consejo Nacional de Ciencia y Tecnología (CONACYT), por la beca proporcionada durante sus estudios de Maestría en Ciencias.

\section{LITERATURA CITADA}

Barbosa, P. 1998. Conservation Biological Control. Academic Press, San Diego. 396 pp.

Bautista-Martínez, N., Chavarín, C. \& López, E. 2011. Primer reporte del enrollador de bandas oblicuas, Choristoneura rosaceana (Harris) (Lepidoptera: Tortricidae), en manzano en el Ejido Vista Hermosa, Ciudad Cuauhtémoc, Chihuahua, México. Acta Zoológica Mexicana (n.s.) 27: 819-824.

Capinera, J. L. 2008. Encyclopedia of entomology (Second Edition). Springer. pp. 3508-3510.

Clifford B. R. \& Taylor R. A. 2008. Bioestadística. Pearson Educación. México. 538 pp.

Cola-Zanuncio, J., Domingues-daSilva, C. A., Rodrigues-deLima, E., Fagundes-Pereira, F., Souza-Ramalho, F. \& Serrao, J. E. 2008. Predation rate of Spodoptera frugiperda (Lepidoptera: Noctuidae) larvae with and without defense by Podisus nigrispinus (Heteroptera: Pentatomidae). Brazilian Archives of Biology and Technology, 51: 121-125.

De Bortoli S. A., Otuka, A. K., Vacari, A. M., Martins, Ma. I. E. G. \& Volpe, H. X. L. 2011. Comparative biology and production costs of Podisus nigrispinus (Hemiptera: Pentatomidae) when fed different types of prey. Biological Control, 58: 127-132.

Jones, V. P., Unruh, T. R., Horton, D. R., Mills, N. J., Brunner, J. F., Beers, E. H. \& Shearer, P. W. 2009. Tree fruit IPM programs in the western United States: the challenges of enhancing biological control through intensive management. Pest Management Science, 65: 1263-1377.

Lagunes T., A., Rodríguez M., J. C. \& De Loera B., J. C. 2009. Susceptibilidad a insecticidas en poblaciones de Artrópodos de México. Agrociencia, 43: 173-196.

Li, S.Y. \& Fitzpatrick, S. 1999. Feeding stimulant added to Bacillus thuringiensis based insecticides enhances activity against Choristoneura rosaceana (Lepidoptera: Tortricidae). The Canadian Entomologist, 131: 451-453.

Ortega-León, G. 1997. Distribución de la subfamilia Asopinae (Hemiptera: Heteroptera: Pentatomidae) para México. Anales Instituto Biología Universidad Nacional Autónoma México, Serie Zoología, 68: 53-89. 
Rider, D. A. 2012. The Heteroptera (Hemiptera) of North Dakota I: Pentatomorpha: Pentatomoidea. The Great Lakes Entomologist, 45: 312-380.

Sarvary, M. A., Nyrop, J. \& Reissing, H. 2007. Assessment of three techniques for measuring natural enemy inflicted mortality of leafroller larvae in commercial orchards. Biological Control, 41: 312-320.

Sarvary, M. A., Nyrop, J. \& Reissing, H. 2010. Effects of natural enemies and host plants in wild and orchard habitats on obliquebanded leafroller (Lepidoptera: Tortricidae) larval survival. Biological Control, 55: 110-117.

Soto, J. 2008. Caracterización molecular de aislamientos de Beauveria bassiana y Metarhizium anisopliae y evaluación de su toxicidad sobre gusano cogollero del maíz Spodoptera frugiperda (J. E. Smith). Tesis de Maestría en Ciencias en Recursos Naturales y Medio Ambiente. IPN. Centro Interdisciplinario de Investigación para el Desarrollo Integral Regional, Unidad Sinaloa, México, 87: 22-30.

Straub, C. S., Finke, D. L. \& Snyder W. E. 2007. Are the conservation of natural enemy biodiversity and biological control compatible goals? Biological Control, 45: 225-237.

Thomas, D. 1992. Taxonomic synopsis of the Asopinae Pentatomidae (Heteroptera) of the western Hemisphere. Entomological Society of America, Lanham, MD. 156 pp.
Tinoco, R. \& Halperin, D. 1998. Poverty, production and health: inhibition of erythrocyte cholinesterase through occupational exposure to organophosphate insecticides in Chiapas, México. Archives of Environmental Health, 53: 29-35.

Torres, J. B., Zanuncio, J. C. \& Moura, M. A. 2006. The predatory stinkbug Podisus maculiventris: biology, ecology and augmentative releases for lepidopteran larval control in Eucalyptus forest in Brazil. Biocontrol News and Information, 27: 1-18.

Universidad de Florida. s/a. Disponible en línea http://entomology. ifas.ufl.edu/creatures/beneficial/podisus_maculiventris.htm, citado en septiembre 2014.

Unruh, T. R., Pfannenstiel, R. S., Peters, C., Brunner, J. F., \& Jones, V. P. 2012. Parasitism of leafrollers in Washington fruit orchards is enhanced by perimeter plantings of rose and strawberry. Biological Control, 62: 162-172.

Vakenti, J. M., Cossentine, J. E., Cooper, B. E., Sharkey, M. J., Yoshimoto, C. M. \& Jensen, L. B. M. 2001. Host-plant range and parasitoids of obliquebanded and three-lined leafrollers (Lepidoptera: Tortricidae). The Canadian Entomologist, 133: 139-146.

Vivan, L. M., Torres, J. B. \& Veiga, A. F. S. L. 2003. Development and reproduction of a predatory stinkbug, Podisus nigrispinus in relation to tow different prey types and environmental conditions. Biocontrol, 48: 155-168. 